\title{
Von Neumann-Lüders projection, and its applicability to EPR experiments
}

\author{
Willem M. de Muynck \\ Theoretical Physics, Eindhoven University of Technology, Eindhoven, the Netherlands
}

\begin{abstract}
Notwithstanding it is well known that von Neumann's projection postulate is inapplicable to most realistic measurement procedures, it keeps haunting the foundations of quantum mechanics. In particular its applicability to EPR experiments is often assumed. In the present contribution this problem is considered from the point of view of a quantum mechanical theory of measurement, allowing a treatment of projection in EPR experiments as a special case of conditional preparation. The conditions are spelled out under which the postulate may be applicable.
\end{abstract}

Keywords: von Neumann-Lüders projection, conditional preparation, EPR

PACS: $02.50 . \mathrm{Cw}$, 03.65.Ca, 03.65.Ta, 03.65.Ud

\section{VON NEUMANN-LÜDERS PROJECTION, MEASUREMENT OR PREPARATION?}

Von Neumann's projection postulate tells us that, if a measurement is performed of observable $\mathbf{A}=\sum_{m} a_{m} \mathbf{P}_{m}$, $\mathbf{P}_{m}=\left|a_{m}\right\rangle\left\langle a_{m}\right|$, and the (non-degenerate) eigenvalue $a_{m}$ is obtained as a measurement result, then the state vector makes a transition

$$
|\psi\rangle \rightarrow\left|a_{m}\right\rangle
$$

from the initial state $|\psi\rangle=\sum_{m} c_{m}\left|a_{m}\right\rangle$ to the corresponding eigenvector $\left|a_{m}\right\rangle$. In case of degeneracy this is generalized to the Lüders projection

$$
|\psi\rangle \rightarrow \frac{\mathbf{P}_{m}|\psi\rangle}{\| \mathbf{P}_{m}|\psi\rangle \|}
$$

$\mathbf{P}_{m}=\sum_{i}\left|a_{m i}\right\rangle\left\langle a_{m i}\right|$ the projection operator on the subspace of Hilbert space spanned by the eigenvectors of $\mathbf{A}$ for which $a_{m i}=a_{m}$.

As a reason for assuming the projection postulate von Neumann ([1] section III.3) refers to the Compton-Simon experiment [2] in which a $\gamma$ photon is scattered by an electron (initially having linear momenta $\mathbf{p}_{p h}$ and $\mathbf{p}_{e}$, respectively), yielding the state

$$
\left|\psi_{\mathrm{CS}}\right\rangle=\int d \mathbf{p}_{e}^{\prime} c\left(\mathbf{p}_{e}^{\prime}\right)\left|\mathbf{p}_{e}^{\prime}\right\rangle\left|\mathbf{p}_{p h}^{\prime}\right\rangle, \mathbf{p}_{p h}^{\prime}=\mathbf{p}_{p h}+\mathbf{p}_{e}-\mathbf{p}_{e}^{\prime}
$$

In the experiment $\mathbf{p}_{p h}$ and $\mathbf{p}_{e}$ are assumed to be known (actually $\mathbf{p}_{e}$ is supposed to be negligibly small compared to the momenta of the $\gamma$ ray photons). Then, by performing in the state (3) a measurement of electron momentum and applying conservation of linear momentum it is possible to obtain from the measurement result $\mathbf{p}_{e}^{\prime}$ the corresponding photon momentum according to $\mathbf{p}_{p h}^{\prime}=\mathbf{p}_{p h}-\mathbf{p}_{e}^{\prime}$. It is concluded that the photon must have this momentum value with certainty, and that the determination of $\mathbf{p}_{e}^{\prime}$ must be accompanied by a transition of the state of the photon to the corresponding momentum eigenstate $\left|\mathbf{p}_{p h}^{\prime}\right\rangle$. This is corroborated by consecutive experiments performed using the scattered photons.

Scattering experiments like the Compton-Simon experiment have been the main tools for testing quantum mechanics during the initial time of its development. As a consequence these experiments could acquire a paradigmatic status with respect to general quantum mechanical measurement, requiring von Neumann projection to be valid for all measurements. This is impossible, however. Thus, it is evident that (1) cannot be applied in a rigorous way to observables having continuous spectra. More importantly, the Compton-Simon experiment has lost its paradigmatic status since many present-day measurement procedures simply do not satisfy (1) or (2). This holds true even for the Stern-Gerlach experiment, which is often presented as satisfying the postulate, the outgoing beams being associated with eigenvectors of the measured angular momentum observable. For two reasons this cannot be true, however. First, 'the atom being in an outgoing beam' does not constitute a measurement. It is a measurement only if it is ascertained which of the beams the atom actually is in. For this reason detectors must be placed in the beams, monitoring the 
presence of the atom. Even if in the Stern-Gerlach experiment by the interaction of the atom with the magnetic field the outgoing states were prepared as eigenvectors of the angular momentum observable, then they can hardly be expected to remain such eigenstates while interacting with a detector which is not designed to leave angular momentum uninfluenced. Second, not even the assumption is warranted that the interaction of the atom with the magnetic field prepares the states of the beams as eigenvectors of the angular momentum observable. It is by now well known ([3], [4] section 8.3.2) -but, unfortunately, widely ignored- that inhomogeneity of the Stern-Gerlach magnetic field is incompatible with the conservation of angular momentum necessary for the assumption to be satisfied. A requirement to satisfy von Neumann projection could be fulfilled only if the magnetic field were homogeneous. Since inhomogeneity of the magnetic field is a necessary condition for the measurement to be effective (be it not without a certain inaccuracy [3]) it turns out that the Stern-Gerlach experiment is an appropriate procedure for the measurement of angular momentum only as a consequence of not satisfying von Neumann projection. This example can be supplemented with many others. For this reason it seems wise not to rely on (1) or (2) when discussing general properties of quantum measurement. That the projection postulate is controversial has been argued before, for instance, by Wigner [5], Ballentine [6] and by Cini and Lévy-Leblond [7].

As a consequence of taking into account a too restricted class of measurements, viz. scattering experiments like the Compton-Simon and the Stern-Gerlach ones, it seemed that preparing the object in some final state was the essence of measurement. The conception of a measurement as a preparation of a microscopic object in a particular final state has become one of the characteristics of the Copenhagen interpretation (Heisenberg [8] section II.2). Nevertheless, it has been realized already a long time ago that von Neumann projection is characterizing only a subset of measurement procedures, referred to as 'measurements of the first kind'. Experiments not preparing the microscopic object in an eigenstate of the measured observable are often referred to as 'measurements of the second kind'.

Confusion of the preparative and determinative aspects of measurement is at the basis of von Neumann's projection postulate (de Muynck [9]). It could arise because no serious theoretical attention was paid to the actual measurement process involved in the interaction of the microscopic object with a (macroscopic) measuring instrument. Admittedly, von Neumann ([1] section VI.3) did consider such interaction. However, his treatment was meant to justify his projection postulate by demonstrating that the latter is consistent with measurement as a quantum mechanical interaction process, rather than to critically examen the feasibility of such processes. Only after it was realized that in measurement procedures the important feature is the preparation of the measuring instrument (yielding a final pointer position $m$, cf. figure 11) rather than the preparation of the microscopic object (represented in figure 1 by the states $\left|\psi_{m}\right\rangle$ ), was it possible to evaluate the role of von Neumann-Lüders projection in quantum mechanical measurement.

\section{QUANTUM MECHANICAL THEORY OF MEASUREMENT, CONDITIONAL PREPARATION, AND VON NEUMANN PROJECTION}

Von Neumann tried to justify the transition (1) by assuming the interaction between object and measuring instrument to induce a transition of the state vector of the system 'object+measuring instrument' according to

$$
|\Psi\rangle=\sum_{m} c_{m}\left|a_{m}\right\rangle|\theta\rangle \rightarrow\left|\Psi_{f}\right\rangle=\sum_{m} c_{m}\left|a_{m}\right\rangle\left|\theta_{m}\right\rangle,
$$

in which $|\theta\rangle$ is the initial state of the measuring instrument, and $\left|\theta_{m}\right\rangle$ are its final pointer states. These latter states are assumed to be mutually orthogonal eigenvectors of the pointer observable $\Theta$. Finding final pointer position $m$ is interpreted as obtaining measurement result $a_{m}$. If (4) is correct, then (1) can be justified by performing in the final state $\left|\Psi_{f}\right\rangle$ a joint measurement of the pointer observable $\Theta$ and an arbitrary observable $B=\sum_{n} b_{n}\left|b_{n}\right\rangle\left\langle b_{n}\right|$ of the object, yielding the joint probability distribution of these compatible observables according to

$$
p\left(m, b_{n}\right)=\mid\left\langle b_{n}\left|\left\langle\theta_{m} \mid \Psi_{f}\right\rangle\right|^{2}=\left|c_{m}\right|^{2}\left|\left\langle b_{n} \mid a_{m}\right\rangle\right|^{2} .\right.
$$

From this the conditional probability $p\left(b_{n} \mid m\right)$ of measurement result $b_{n}$, given the final pointer position $m$, is found as

$$
p\left(b_{n} \mid m\right)=\frac{p\left(m, b_{n}\right)}{p(m)}=\left|\left\langle b_{n} \mid a_{m}\right\rangle\right|^{2} .
$$

From the arbitrariness of $B$ it follows that the state of the object, conditional on pointer position $m$, must be given by $\left|a_{m}\right\rangle$, as required by (1). 


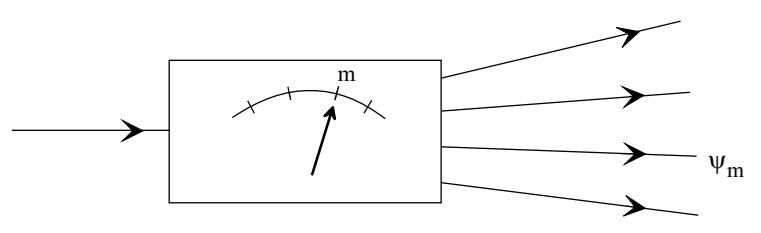

FIGURE 1. Determinative and preparative aspects of measurement.

Unfortunately, the measurement scheme (4) refers only to measurements of the first kind. In order to take into account the possibility of measurements of the second kind the scheme (4) should be replaced by

$$
|\Psi\rangle=\sum_{m} c_{m}\left|a_{m}\right\rangle|\theta\rangle \rightarrow\left|\Psi_{f}\right\rangle=\sum_{m} c_{m}\left|\psi_{m}\right\rangle\left|\theta_{m}\right\rangle
$$

in which the vectors $\left|\psi_{m}\right\rangle$ are normalized but in general not mutually orthogonal. What is the final state of the object is determined by the interaction between object and measuring instrument. In general this interaction disturbs the object so as to end up, conditional on pointer position $m$, in a state $\left|\psi_{m}\right\rangle$ different from $\left|a_{m}\right\rangle$, even if this was the object's initial state. Analogously to (5) and (6) it is found that (1) should be replaced by the transition

$$
|\psi\rangle \rightarrow\left|\psi_{m}\right\rangle \text {. }
$$

Hence, in general von Neumann's projection postulate is not satisfied (nor is the Lüders one).

The theory of measurement allows to distinguish between determinative and preparative aspects of measurement (cf. figure 1). The determinative aspect refers to the knowledge on the initial state to be obtained by determining the final pointer position of a measuring instrument. The preparative aspect refers to what has become of the object after the measurement. These are completely different features, being related by von Neumann's projection postulate in an untoward way. Transitions like (1) and (8) refer to conditional preparations, preparing the object in state $\left|a_{m}\right\rangle$ or $\left|\psi_{m}\right\rangle$ if measurement result $m$ is obtained. If the object is not absorbed by the measuring instrument, then it is possible in principle to perform a consecutive experiment while selecting outgoing objects on the basis of pointer positions $m$, so as to be sure to have conditionally prepared states. The idea that von Neumann-Lüders projection is a general property of measurement stems from a confusion of the determinative and preparative aspects of quantum measurement, transitions like (1) and 2) describing preparation procedures rather than measurements.

\section{VON NEUMANN-LÜDERS PROJECTION AND EPR}

It is evident that, if applicable at all to EPR [10], we should consider Lüders projection rather than von Neumann's, since observable $\mathbf{A}^{(1)}$ of particle 1 should actually be considered as an operator $\mathbf{A}^{(1)} \mathbf{I}^{(2)}$ on a two-particle space, which has degenerate eigenvalues even if the eigenvalues of $\mathbf{A}^{(1)}$ are non-degenerate (which shall be assumed here). Taking the initial EPR state according to

$$
\left|\psi_{\mathrm{EPR}}\right\rangle=\sum_{m} c_{m}\left|a_{m}^{(1)}\right\rangle\left|a_{m}^{(2)}\right\rangle
$$

we get

$$
\mathbf{P}_{m}=\left|a_{m}^{(1)}\right\rangle\left\langle a_{m}^{(1)}\left|\sum_{m^{\prime}}\right| a_{m^{\prime}}^{(2)}\right\rangle\left\langle a_{m^{\prime}}^{(2)}|=| a_{m}^{(1)}\right\rangle\left\langle a_{m}^{(1)}\right| \mathbf{I}^{(2)},
$$

in the state $\left|\psi_{\mathrm{EPR}}\right\rangle$ yielding a Lüders projection (2) according to

$$
\left|\psi_{\mathrm{EPR}}\right\rangle \rightarrow\left|a_{m}^{(1)}\right\rangle\left|a_{m}^{(2)}\right\rangle
$$

This result is in agreement with our experience with respect to measurements consecutively performed on particle 2. However, it does not take into account a possible disturbance of particle 1 by the measurement of $\mathbf{A}^{(1)}$ actually carried 
out. Indeed, taking such a disturbance into account, application of the theory of measurement to EPR yields transitions (7) and (8) according to

$$
|\Psi\rangle=\sum_{m} c_{m}\left|a_{m}^{(1)}\right\rangle\left|a_{m}^{(2)}\right\rangle|\theta\rangle \rightarrow\left|\Psi_{f}\right\rangle=\sum_{m} c_{m}\left|\psi_{m}^{(1)}\right\rangle\left|a_{m}^{(2)}\right\rangle\left|\theta_{m}\right\rangle
$$

and

$$
\left|\psi_{\mathrm{EPR}}\right\rangle \rightarrow\left|\psi_{m}^{(1)}\right\rangle\left|a_{m}^{(2)}\right\rangle
$$

respectively. Hence, it is allowed to apply von Neumann projection to particle 2. But, as before, the measurement interaction prevents the postulate in general to be satisfied by the particle that has interacted with the measuring instrument.

Comparing (3) and (9) a close similarity can be observed between the EPR and Compton-Simon experiments, the latter serving von Neumann as an incentive to assume his projection postulate (1). In both cases it is the object not interacting with the measuring instrument that is assumed to be subjected to projection, the actual measurement being carried out on another object. In both cases the object not interacting with the measuring instrument is not actually subjected to a measurement, but it is prepared in a quantum mechanical state $\left(\left|\mathbf{p}_{p h}^{\prime}\right\rangle\right.$ and $\left|a_{m}^{(2)}\right\rangle$, respectively), which can act as an initial state in a new experiment (not necessarily a measurement) performed with the photons c.q. particles corresponding to results $\mathbf{p}_{e}^{\prime}$ c.q. $a_{m}^{(1)}$ obtained from measurements on correlated objects. From this it is evident that, although von Neumann projection may not be applicable to measurement, it may yield a description of conditional preparation. Indeed, conditional preparation is a widely used experimental procedure for preparing an object in a welldefined state, unfortunately often referred to as a 'measurement', for instance, as a 'predictive measurement' (Kemble [11] section 41). Von Neumann-Lüders projection is applicable only as far as the measurement does not disturb the object.

\section{GENERAL THEORY OF CONDITIONAL PREPARATION}

The method of conditional preparation can easily be extended to less simplistic, and possibly more realistic measurement procedures than considered in sections $\mathbf{2}$ and $\mathbf{3}$. Indeed, the initial states of object and measuring instrument may be represented by density operators $\rho^{(o)}$ and $\rho^{(a)}$, respectively. Let the measurement interaction yield as its final state

$$
\rho_{f}=\mathbf{U} \rho^{(o)} \rho^{(a)} \mathbf{U}^{\dagger}, \mathbf{U}=\exp -i \mathbf{H} T
$$

$\mathbf{H}$ the interaction Hamiltonian, $T$ the interaction time. Then the detection probabilities are given by

$$
p(m)=\operatorname{Tr}_{o a} \rho_{f} \mathbf{E}_{m}^{(a)}, \mathbf{E}_{m}^{(a)}=\sum_{i}\left|\theta_{m i}\right\rangle\left\langle\theta_{m i}\right|,
$$

in which the $\left|\theta_{m i}\right\rangle$ are the degenerate eigenvectors of the pointer observable representing the pointer's microscopic structure at pointer position $m$.

The conditionally prepared state can be found, as before, by contemplating in the final state a joint measurement of the pointer observable and an arbitrary observable of the object, yielding joint probabilities

$$
p(m n)=\operatorname{Tr}_{o a} \rho_{f} \mathbf{E}_{m}^{(a)} \mathbf{F}_{n}^{(o)} .
$$

The conditionally prepared states $\rho_{f m}^{(o)}$ can now be found as those object states for which the conditional probabilities $p(n \mid m)$, related to $p(m n)$ and $p(m)$ according to $p(m n)=p(n \mid m) p(m)$, satisfy

$$
p(n \mid m)=\operatorname{Tr}_{o} \rho_{f m}^{(o)} \mathbf{F}_{n}^{(o)}
$$

As a consequence of the arbitrariness of $\left\{\mathbf{F}_{n}^{(o)}\right\}$ it straightforwardly follows from (16) and (17) that

$$
\rho_{f m}^{(o)}=\frac{\operatorname{Tr}_{a} \rho_{f} \mathbf{E}_{m}^{(a)}}{\operatorname{Tr}_{o a} \rho_{f} \mathbf{E}_{m}^{(a)}}
$$


As demonstrated by de Muynck ([4] section 3.3.4) this derivation applies even if the measurement is a generalized one in which the probabilities (15) correspond to a positive operator-valued measure $\left\{\mathbf{M}_{m}=\operatorname{Tr}_{a} \rho^{(a)} \mathbf{U}^{\dagger} \mathbf{E}_{m}^{(a)} \mathbf{U}\right\}$. In particular, if $\mathbf{M}_{m}=\sum_{m^{\prime}} \lambda_{m m^{\prime}}\left|a_{m^{\prime}}\right\rangle\left\langle a_{m^{\prime}}\right|, \lambda_{m m^{\prime}} \geq 0, \sum_{m} \lambda_{m m^{\prime}}=1$, the measurement is a nonideal measurement of the standard observable $\mathbf{A}$ (de Muynck [4] section 7.6.5). This is satisfied if

$$
\operatorname{Tr}_{o a} \mathbf{U}\left|a_{m^{\prime}}\right\rangle\left\langle a_{m^{\prime \prime}}\right| \rho^{(a)} \mathbf{U}^{\dagger} \mathbf{E}_{m}^{(a)}=\lambda_{m m^{\prime}} \delta_{m^{\prime} m^{\prime \prime}} .
$$

Such nonideal measurements are encountered in many practical applications of quantum measurement theory (e.g. de Muynck [4] chapter 8).

The theory of conditional preparation can straightforwardly be applied to the EPR experiment by specifying the object (o) to consist of 2 particles (labeled 1 and 2, respectively) and taking $\mathbf{F}_{n}^{(o)}=\mathbf{F}_{n_{1}}^{(1)} \mathbf{F}_{n_{2}}^{(2)}$. Analogously to (18) we find as the conditionally prepared state of the two-particle system

$$
\rho_{f m}^{(12)}=\frac{\operatorname{Tr}_{a} \rho_{f} \mathbf{E}_{m}^{(a)}}{\operatorname{Tr}_{12 a} \rho_{f} \mathbf{E}_{m}^{(a)}} .
$$

Let us apply this to the EPR state $\left|\psi_{\mathrm{EPR}}\right\rangle=\sum_{m} c_{m}\left|a_{m}^{(1)}\right\rangle\left|a_{m}^{(2)}\right\rangle$ (9), taking the measurement to be of observable $\mathbf{A}^{(1)}$ of particle 1 only. Since the interaction operator $\mathbf{U}$ does not depend on the particle 2 variables we have $\left[\mathbf{U},\left|a_{m^{\prime}}^{(2)}\right\rangle\left\langle a_{m^{\prime \prime}}^{(2)}\right|\right]_{-}=\mathbf{O}$. From (20) it then follows that

$$
\rho_{f m}^{(2)}=\operatorname{Tr}_{1} \rho_{f m}^{(12)}=\frac{1}{p(m)} \sum_{m^{\prime} m^{\prime \prime}} c_{m^{\prime}} c_{m^{\prime \prime}}^{*}\left|a_{m^{\prime}}^{(2)}\right\rangle\left\langle a_{m^{\prime \prime}}^{(2)}\left|\operatorname{Tr}_{1 a} \mathbf{U}\right| a_{m^{\prime}}^{(1)}\right\rangle\left\langle a_{m^{\prime \prime}}^{(1)}\right| \rho^{(a)} \mathbf{U}^{\dagger} \mathbf{E}_{m}^{(a)}
$$

If we take in (19) particle 1 for object $o$, then for a nonideal measurement of $\mathbf{A}^{(1)}$ we obtain from (19) and (21)

$$
\rho_{f m}^{(2)}=\sum_{m^{\prime}} \frac{\lambda_{m m^{\prime}}\left|c_{m^{\prime}}\right|^{2}}{\sum_{m^{\prime \prime}} \lambda_{m m^{\prime \prime}}\left|c_{m^{\prime \prime}}\right|^{2}}\left|a_{m^{\prime}}^{(2)}\right\rangle\left\langle a_{m^{\prime}}^{(2)}\right|
$$

demonstrating that von Neumann projection can only be satisfied if the measurement of $\mathbf{A}^{(1)}$ is an ideal one (for which $\lambda_{m m^{\prime}}=\delta_{m m^{\prime}}$ ). In particular, a detector efficiency smaller than 1 is causing the conditionally prepared state to deviate from the von Neumann ideal.

\section{ACKNOWLEDGMENTS}

The author likes to thank the Faculty of Applied Physics of Eindhoven University of Technology, and in particular professor Thijs Michels, for providing facilities that made this work possible. Thanks are due also to Andrei Khrennikov for his invitation to publish the paper in the Proceedings of FPP-5.

\section{REFERENCES}

1. J. von Neumann, Mathematische Grundlagen der Quantenmechanik, Springer, Berlin, 1932; or, Mathematical foundations of quantum mechanics, Princeton Univ. Press, 1955.

2. A.H. Compton and A.W. Simon, Phys. Rev. 25, 306-314 (1925).

3. H. Martens and W.M. de Muynck, Journ. Phys. A: Math. Gen. 26, 2001 (1993).

4. Willem M. de Muynck, Foundations of quantum mechanics, an empiricist approach, Fundamental theories of physics, vol. 127, Kluwer Academic Publishers, Dordrecht, Boston, London, 2002.

5. E.P. Wigner, Amer. Journ. of Phys. 31, 6 (1963).

6. L.E. Ballentine, Found. of Phys. 20, 1329 (1990).

7. M. Cini, J.-M. Lévy-Leblond eds., Quantum theory without reduction, Adam Hilgers, Bristol and New York, 1990.

8. W. Heisenberg, The physical principles of quantum theory, Dover Publications, Inc., 1930.

9. W.M. de Muynck, Found. of Phys. 30, 205 (2000).

10. A. Einstein, B. Podolsky, and N. Rosen, Phys. Rev. 47, 777 (1935).

11. E.C. Kemble, The Fundamental Principles of Quantum Mechanics, McGraw-Hill Book Company, Inc., New York, 1937. 